\title{
COMBINED MODELLING OF MULTIPLE TRANSPORTATION INFRASTRUCTURE WITHIN 3D CITY MODELS AND ITS IMPLEMENTATION IN CITYGML 3.0
}

\author{
C. Beil *, T. H. Kolbe \\ Chair of Geoinformatics, Technical University of Munich, Germany - (christof.beil, thomas.kolbe)@tum.de
}

Commission VI, WG VI/4

\begin{abstract}
KEY WORDS: CityGML, Multimodal Transportation, Road, Railway, Waterway, Pedestrian, 3D City Models, Digital Twin
\end{abstract}
\begin{abstract}
:
The development of digital urban twins has led to efforts of multiple cities to gather detailed and highly accurate data on many parts of urban environments, including road and railway infrastructure as well as areas used by pedestrians. This paper presents concepts for representing and segmenting this data semantically, geometrically and topologically ensuring usability for various multimodal applications in the context of digital 3D city models. First, methods for modelling multiple transportation types of several standards such as OpenDRIVE, GDF and INSPIRE are examined, including a discussion of different types of information integration such as functional and topographical representations. Then, concepts proposed to the CityGML Standards Working Group for the CityGML 3.0 Transportation model are presented. This includes detailed methods for modelling multiple transportation modes within a common city model while avoiding redundant geometric representations. A section / intersection concept in combination with links used to model these relations is described. Linear as well as areal models of level crossings, pedestrian crosswalks or areas shared by multiple transportation infrastructure at the same time (e.g. a tramway within a road) are presented. Subsequently, examples and applications that benefit from accurate representations of multiple transportation types are described.
\end{abstract}

\section{INTRODUCTION}

In the context of digital twins and smart cities, semantic 3D city models, along with the most commonly used OGC standard CityGML, provide an opportunity to represent real world scenarios in a consistent and standardised way. While models of buildings in level of detail (LoD) 2 have become very common, this has also led to developments in other thematic areas such as roads or railways. Beil \& Kolbe (2017) and Labetski et al. (2018) showed how streetspace can be represented as part of detailed semantic 3D city models and presented various applications. Until now there are very few examples for concepts, let alone actual demos, of (combined) road, pedestrian, railway and waterway models within digital 3D city models. Several cities (including Munich or Vienna) have started gathering data for the development of digital twins to be used for simulations and analyses of urban environments (Batty 2018). Data acquisitions with mobile mapping systems can deliver highly accurate point clouds of buildings as well as streets, city furniture or railways. A very important next step in this process will be to use this data for semantically segmented and topologically connected representations of cities and landscapes in order to be usable for a large variety of applications. Gröger \& Plümer (2011) showed the importance of geometric, semantic and topologic consistency within 3D city models. This paper focusses on combined modelling of multiple transportation types, providing concepts for non-redundant geometric and semantic representations in order to ensure a consistent and integrated city model. This requires standards such as the international OGC standard CityGML, used for city and landscape modelling. Recently, a proposal for a revised Transportation Model for the new version of CityGML 3.0 has been presented in the CityGML Standards Working Group. It contains concepts for combined modelling of multiple transportation modes. This paper presents and discusses the new Transportation Model.

\section{MOTIVATION FOR COMBINED MODELLING OF MULTIPLE TRANSPORTATION INFRASTRUCTURE}

While there are several approaches to modelling streetspace, most standards focus on a specific type of transportation (e.g. the representation of roads used by cars). Other types of transportation are often neglected or only supplementary to the main transportation type. However, in order to express connections between different transportation modes, different types of transportation need to be represented and integrated within a common city model (Gonzales et al. 2010). Transportation networks, especially within large cities, do not only consist of road traffic but also include other transportation types such as bikes, pedestrians, railways, trams or sometimes even waterway traffic on rivers and canals. These different transportation forms often do not just coexist next to each other but often directly interact both functionally and topographically. Thus, modelling approaches can be distinguished regarding their functional and topographical representation. Functional representations integrate different transportation modes (such as roads, footpaths or railways) by combining multiple graphbased networks including information on traffic rules such as turning restrictions or traffic control (e.g. traffic light system). Topographical representations on the other hand allow a nonredundant geometric and semantic modelling of multiple transportation infrastructure by representing the spatial occupancy by individual objects (length, width, height, overlap). Level crossings of road and railway, pedestrian crosswalks or trams permanently sharing parts of a road surface with cars and other vehicles are very common. Modelling these multimodal transportation relations within 3D city models in a non-redundant and consistent way can be difficult. Nonetheless, it is essential in order to represent realistic real world scenarios usable for different applications, especially beyond navigation. Different geometric representations with linear graph networks, areal surface models or volumetric spaces of the same scenario

\footnotetext{
* Corresponding author
} 
should be possible depending on the intended application. This adds another aspect to this topic since different representations of the same multimodal traffic scenario should be consistent with one another. It should be supported to represent areas and structures such as roads, railways, bikeways or sidewalks used by different traffic members (simultaneously or alternately). Beil \& Kolbe (2017) and Labetski et al. (2018) listed a variety of applications for detailed streetspace models. Some of these applications would also benefit from consistent and integrated representations of combined transportation infrastructure. This can be important in the context of combined vehicle and pedestrian simulations (Schwab et al. 2020). Pedestrian crosswalks, for example, are part of road surfaces that can be used by cars as well as pedestrians. Modelling this without using redundant geometrical representations can be challenging. Connections between various transportation types can be relevant for (multimodal) navigational purposes to indicate if traffic members can switch between different transportation systems. In some scenarios different transportation types may intersect, without traffic members being able to change systems. A level crossing for example may share areas used by roads as well as railways, while traffic members obviously cannot switch between the two systems. Virtual testing scenarios in the context of autonomous driving rely on detailed semantic and geometric information of streetspace (Schwab \& Kolbe 2019). This requires information on areas used by different traffic members as well as their topological connections. Land management and urban planning applications can also benefit from detailed information on streetspace with multiple transportation types. Tamminga (2019) presented concepts for modelling transportation infrastructure in the context of (multimodal) traffic simulations. Gnatz (2018) created transportation models for tram simulators using the software CityEngine (Müller et al. 2006). Figure 1 shows that intersections of different transportation systems such as streets, trams or pedestrian crosswalks can get complex. Concepts for segmenting such scenarios for areal as well as linear models in the context of city models are discussed in this paper.

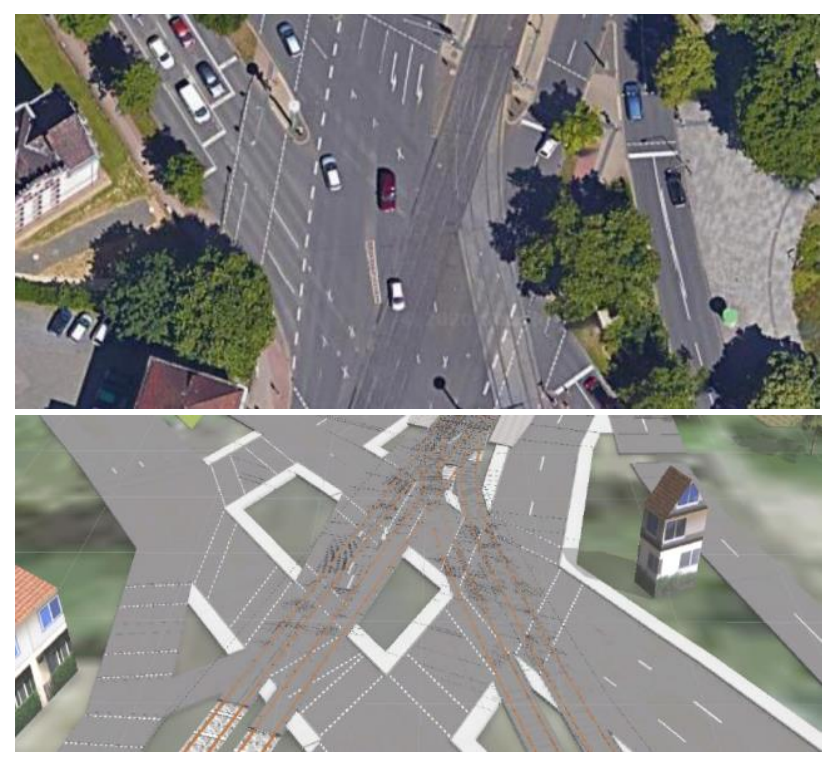

Figure 1. Complex intersection of roads and trams. GoogleEarth (top), model generated with CityEngine (bottom) (Gnatz 2018)

First, standards relevant in the context of streetspace modelling are examined and discussed with regard to their potential for combined modelling of multiple transportation types.

\section{MODELLING APPROACHES OF DIFFERENT STANDARDS}

Beil \& Kolbe (2017) and Labetski et al. (2018) presented relevant standards focusing on streetspace modelling in general. In this paragraph, different standards related to transportation are examined specifically with regard to the modelling of combinations of multiple transportation types as well as multimodal scenarios. Additionally, relations between areas used by various transportation types such as level crossings, pedestrian crosswalks or tramways within the same road surfaces are also examined. While there are examples for modelling multiple transportation infrastructure, that are not based on a standard, standards are useful for generating consistent and integrated models. Software packages are often tailored towards standardised data models and thus ensure its usability. Non-standard models are therefore excluded from the following discussion.

\subsection{Geographic Data Files}

Geographic Data Files (GDF) is a standard mostly used for vehicle navigation purposes. The current version GDF5.0 was published in 2011 (ISO 14825 2011). Features defined within so-called Feature Catalogues and categories for several thematic themes including Road and Ferry, Railway, Road Furniture or Public Transport. These themes can be represented in different levels. Topological primitives such as nodes and edges are part of Level-0. Level-1 is defined as simple feature representation, while Level-2 objects are aggregations of simple features. Road networks can be represented in Level-1 and Level-2. Simple Features such as Road Element or Junction in Level-1 can be aggregated to form complex features such as a Road, Intersection or Interchange in Level-2. Road elements are always represented with line features, while junctions are always represented by point features. Enclosed Traffic Areas (squares or parking lots) are represented by areal features. Junctions can be further specified with a Junction Type attribute. This includes Railway Crossings as well as crossings between a road and a dedicated pathway for vehicles such as trams, trains or busses defined as Fixed Guideway Vehicle Crossings. Railways can only be modelled with linear Railway Elements and points representing Railway Element Junctions in Level-1. A Level-2 representation is not available. Pedestrian Crossings can be represented explicitly and are modelled as sub-class of Road Furniture Features with either point, line or area geometries depending on size and defined accuracy.

\subsection{OpenDRIVE}

OpenDRIVE is an open XML-based data format for highly detailed descriptions of street networks. Since 2018 the standard is managed by the Association for Standardization of Automation and Measuring Systems (ASAM). Main field of application are driving simulations used by automobile manufacturers including AUDI and BMW. The current format specification version 1.6 was published in 2020 (ASAM 2020). Roads are modelled parametrically using a reference line (Track), which defines the basic geometry. Multiple Roads can be linked to each other directly or via Junctions. Different coordinate systems can be used including a Track System containing the position along the reference line (s), a lateral position (t) and a vertical value (h). Multiple Lane Sections may be defined along a reference line. Lanes of a Road are unique per lane section, in sequence, starting from 0 on the reference line, ascending to the left (positive t-direction) and descending to the right (negative t-direction). The total number of lanes is 
not limited and the reference line itself is defined as lane 0 . These lanes can be of several types including driving, biking, sidewalk, tram, rail, taxi or bus. OpenDRIVE is mainly designed for detailed parametric descriptions of streets, nonetheless it includes concepts for modelling railroad elements, especially in cases where roads and rails meet. In addition to describing lane types of a Road (e.g. as rail), Railroad Elements can also be represented with a reference line (Railroad Track) of their own. It is mandatory to use one discrete Track per direction for railroad lines with multiple parallel Tracks instead of adding railroad lanes to an existing track. Additionally, it is recommended to provide individual reference lines for rail and road objects even if they share identical areas. Similar to Junctions, Railroad Switches can be used to represent connections between Railroad Elements. Railroad lines may also contain Railroad Stations with Platforms. Several Railroad Tracks may reference the same Platform. In order to be able to describe common 3D objects which have a reference to a road, such as trees or walls, so called Objects can be represented. This explicitly includes Crosswalks, which are modelled using so called CornerRoad points defining an Objects' outline in Road coordinates (s, t, h).

\subsection{LandInfra}

LandInfra is an OGC standard that defines concepts for land and civil engineering infrastructure facilities (Gruler et al. 2016). Most relevant parts of the standard with regard to multimodal transportation modelling are Alignment, Road and Railway. A Road consists of several RoadElements, which are further specified with a roadElementType attribute including values such as sidewalk and bikePath. The same concept applies for Railways and RailwayElements. Interactions between different transportation types as well as other transportation types such as tramways or busways are not specifically addressed.

\subsection{INSPIRE}

The initiative Infrastructure for Spatial Information in Europe (INSPIRE) aims for interoperability of spatial data and services from different sources across the European Community and includes conceptual data models for transportation networks (INSPIRE 2014). This includes concepts for five distinct transportation themes: Road, Rail, Water, Air-transport and Cableways including connection between the different types. These themes are based on the INSPIRE Generic Network Model (GNM) that relies on multiple ISO 191XX geographic information standards. The GNM includes a network connection mechanism, object referencing to support the reuse of information (e.g. to avoid redundant geometry representation) and a linear referencing system. Network elements are handled as nodes, links, aggregated links, areas and points. Three types of geometry are possible: a) (topographic) area objects, b) centerlines or c) point objects. Geometries a) and b) can be used for alternative representation of the same real world object, while points (apart from network nodes) are only used for marker posts used for indicating distances along a route.

INSPIRE is one of only few standards that explicitly addresses concepts for representing multimodal transportation relations. Multimodal connections between different transportation networks (e.g. Rail and Road) can be modelled by linking (cross-referencing) spatial objects in order to support connectivity. This is achieved by providing a Network Connection class to establish cross-border connectivity between transportation networks of different countries. This Network Connection object can also be used to represent multimodal connections by linking different transportation networks to a Network Connection object defined as multimodal connection by a ConnectionTypeValue attribute. While several transportation network elements such as nodes, links and areas can participate in multimodal connections, the use of nodes is recommended in order to ensure a more accurate positioning of the connection between two networks. With this concept, nodes in different Transportation Networks can be connected. These nodes may but do not have to share the same location. This way a RailwayStationNode may be connected to a RoadNode even though these nodes may be spatially separated. This is beneficial in order to represent a possibility for transport media (e.g. people) to change from one transportation type to another. The object referencing system in combination with Network Connections can be used to represent multimodal traffic relations such as level crossings or pedestrian crossings

\subsection{OpenStreetMap}

OpenStreetMap (OSM) is a project for collecting user generated open (geo) data available for web viewing and download (Haklay \& Weber 2008). Features are identified by so called keys. Additionally, a number of attributes can be assigned to each feature. Some of the most important features in the context of (multimodal) transportation modelling are Highway (used for all kinds of roads, including streets, footpaths or cycle tracks), Railway, Aeroway, Route but also transportation sub-features of Amenity (facilities used by visitors and residents; e.g. a bus station). While this wide range of features allows to model many different transportation forms, most of these objects (including highway and railway features) are represented with linear geometries only. Availability and accuracy of the data depends on the geographic location and how the data was collected. Multimodal transportation scenarios such as level crossing can be represented with nodes but are a subtype of Railway and thus not connected to Highways. A crossing as subtype of Highway is defined as crosswalk used by pedestrians and represented with point geometries. Logical relationships between objects can be modelled using Relations.

\subsection{Industry Foundation Classes (IFC)}

The currently valid standard Industry Foundation Classes 4.2 (IFC4.2) was published in 2018 (ISO 16739-1 2018). IFC is a Building Information Modelling (BIM) data exchange format used for the representation of constructed objects. While the current standard is mainly designed for representing buildings, it offers limited possibilities for modelling transportation infrastructure. IFCAlignment is used for modelling linear construction structures such as roads or railways. Currently several projects, including IFCRoad, IFCRail, IFCTunnel or IFC Ports \& Waterways, are introducing new concepts. These concepts will be integrated into the next version IFC4.3, which is scheduled to be released in 2020. This will also contain concepts for modelling interactions of multiple transportation types such as level crossings.

\subsection{CityGML2.0}

CityGML is one of the most widely used standard for 3D city modelling. The current version CityGML2.0 was published in 2012 (Gröger et al. 2012). The standard contains concepts for many different thematic parts of a city including buildings, vegetation and transportation in different levels of detail (LoD). Transportation objects are modelled as TransportationComplex 
with sub-level features Road, Railway, Track and Square. Linear representation is used to represent LoD0 models. In LoD1 areal models representing the entire width of a transportation object are possible. Starting from LoD2, these objects can be further divided semantically into AuxiliaryTrafficAreas (describing further elements of a road such as kerbstones or green areas) and TrafficAreas (areas where the actual traffic takes place). Each transportation object can also be specified using class, function and usage attributes defined within extensive code lists. This way, many different transportation types, including footpaths, bikeways, tramways or busways can be represented. While this allows for representing many different transportation types, intermodal links between different transportation modes are not provided. A level crossing for example can be represented as a TrafficArea that is part of a Road and a Railway object simultaneously. The same applies for pedestrian crossings or tramways within a Road.

\subsection{Summary and comparison of the standards presented}

Depending on the main purpose and application of a specific standard it may not be necessary to represent multiple transportation types and their relations. However, as explained in chapter 2 , in the context of $3 \mathrm{D}$ city modelling this is important for ensuring accurate and non-redundant representations. Table 1 summarizes which transportation types can be represented using a specific standard. The list also includes CityGML version 3.0, which is explained in more detail in chapter 4. A detailed explanation on each cell is provided on a corresponding Wiki page (http://go.tum.de/863516). Modelling concepts such as the dimension of the coordinate space used for representing transportation objects or the availability of linear, areal or parametric representations are indicated. Beil \& Kolbe (2017) explained differences between these representation types in detail. While some standards only allow functional representations, others provide possibilities for combined functional and topographical representations. Graph based functional integration can be achieved in different degrees of complexity. Basic functional integration of multiple transportation types (allowing simple routing applications) is provided by all standards $(+)$. While CityGML 3.0 additionally provides a predecessor / successor concept $(++)$, standards such as GDF and OpenDRIVE include detailed specifications on aspects such as speed limits, turning restrictions or traffic control, while also integrating traffic signs and traffic lights $(+++)$. Additionally, multimodal topologic connections are necessary for route planning or navigational purposes, where switching between different transportation types is considered. Cells are coloured green if Transportation types are available either as classes or with attributes further describing a more general class. For example: Tramways are not modelled as a specific class in CityGML2.0 but can be represented using a Railway object with a function attribute value "Tram". Subways can be represented using a combination of CityGML Railway and Tunnel modules. Orange cells indicate that there is no explicit concept in the standard to represent these transportation types. Yellow cells indicate that this object could be represented by using some kind of workaround. For example: CityGML2.0 does provide function attributes for TransportationComplexes called "Ferry" or "Waterway", however an explicit class for "Waterways" is not available. Additionally, table 1 indicates if relations between multiple transportation types such as level crossings of road and rail objects, pedestrian crossings or tramways using part of road surfaces can be represented with the respective standard. Objects typically used for multimodal connections of different transportation types such as railway stations, bus stands or subway entrances are also listed. In summary most standards presented are able to represent multiple transportation types. Different transportation types are often taken into account by using multiple attribute values indicating an intended function of specific parts of a transportation network. INSPIRE also uses specific classes to model transportation relations such as pedestrian or level crossings. Modelling multimodal connections for representing switches between transportation modes is available for a limited number of standards. While most standards use a linear representation for modelling transportation infrastructure, multimodal topologic connections between these networks are not always possible. Topographical representations using nonredundant areal objects are available for a limited number of standards only. CityGML 3.0 as well as IFC4.3 will feature detailed concepts for representing multiple transportation modes and interactions between these types. CityGML 3.0 offers elaborate concepts for detailed functional as well as topographic representations of combined transportation infrastructure models for multiple transportation types. Thus, concepts of this international OGC standard are presented in the next chapter.

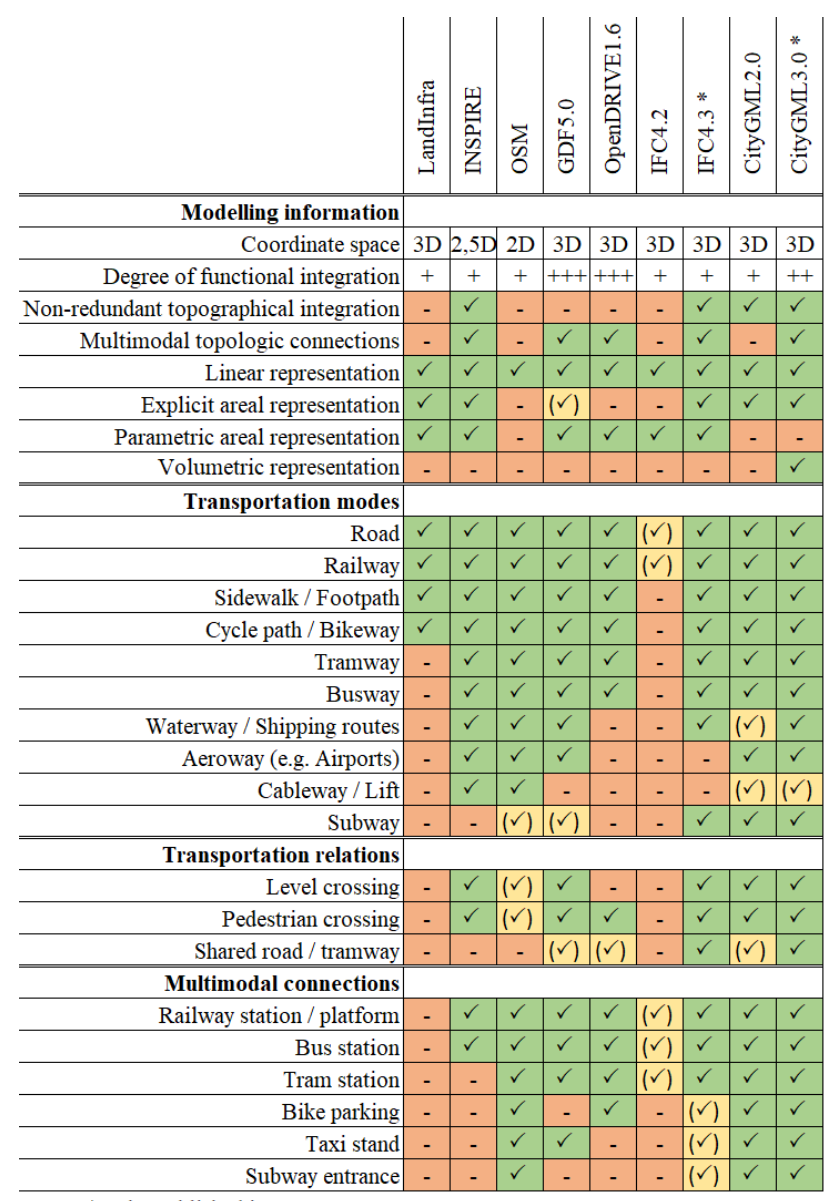

* to be published in 2020

Table 1. Support of transportation modes and their combinations in different standards 


\section{MODELLING MULTIPLE TRANSPORTATION TYPES WITH CITYGML 3.0}

Beil \& Kolbe (2017) as well as Labetski et al. (2018) addressed limitations of the CityGML 2.0 Transportation model and proposed several improvements. Tamminga (2019) addressed shortcomings of CityGML 2.0 with regard to multimodal traffic simulations, focusing on functional, but not topographical integration. For explanations of new CityGML 3.0 concepts, please refer to Kutzner et al. (2020). The proposed conceptual model can be found on the public GitHub repository for the Open Geospatial Consortium (OGC) CityGML Standards Working Group (SWG) ${ }^{1}$. This includes a revised Transportation Model. First, essential concepts of this revised CityGML 3.0 Transportation model are explained. Please note, that the following paragraph focuses on concepts relevant for combined modelling of multiple transportation modes within digital 3D city models.

\subsection{Proposed CityGML 3.0 Transportation Model}

The main class is called AbstractTransportationSpace, which is divided into 5 subclasses called Road, Railway, Square, Track and newly introduced Waterway. Except for Square each of these subclasses can be semantically and geometrically split into Sections and Intersections. Similar to concepts presented in the CityGML2.0 standard, TrafficAreas and AuxiliaryTrafficAreas can be used to further semantically segment Sections and Intersections. Kutzner et al. (2020) introduce a concept to represent real world objects with a volumetric extend as Spaces. Correspondingly, TrafficSpaces and AuxiliaryTrafficSpaces are presented. TrafficAreas represent the ground surface of a TrafficSpace. Transportation objects can be modelled with a linear, areal or volumetric representation. In order to express different levels of complexity and detail of individual transportation objects, three levels of granularity are introduced. Granularity level 'area' is used for representing each Section by only one TrafficSpace, in granularity level 'way' each traffic way (e.g. carriageway used by vehicles or sidewalks used by pedestrians) is modelled. The highest level of granularity called 'lane' uses lane level accuracy by representing each lane individually. Note, that these different levels are not distinguished by CityGML's LoD concept, because in CityGML 3.0 LoDs only refer to the spatial representation and no longer to the semantic differentiation (Löwner et al. 2016, Kutzner et al. 2020). A new predecessor / successor relation allows the representation of connections between multiple TrafficSpaces to indicate allowed movements within a transportation network. This concept is especially important for multimodal and intermodal transportation and can be applied to areal and linear models. An additional attribute called TrafficDirection indicates the traffic flow direction relative to the directionality of linear representations of TrafficAreas. CityGML 3.0 is based on the ISO 191XX series of geographic information. Geometries used in CityGML 3.0 are based on ISO 19107. GM_MultiSurface geometries are used for areal representations of transportation objects. Linear structures are represented using GM_MultiCurve geometries. In contrast to GM_Geometric Complex geometries used in CityGML2.0, GM_MultiCurves can - but do not have to - have shared point objects (nodes) at intersecting lines. This way, intersecting road and railway networks do not have to have nodes, thus preventing potential misinterpretations in the context of routing or navigational applications. Several other thematic modules, such as Tunnel or Bridge, can also be relevant especially when under- or overpasses of roads or

${ }^{1}$ https://github.com/opengeospatial/CityGML-3.0CM railways should be represented accurately. This also requires a 3D coordinate space. Due to limited space and because many applications (such as testing autonomous driving systems) rely on highly accurate models, the following examples demonstrate these concepts for several scenarios with lane level granularity only. The presented concepts also apply for sub-surface transportation infrastructure.

\subsection{Modelling multiple Transportation modes within the same top-level feature}

TrafficAreas or TrafficSpaces can be used by multiple transportation types simultaneously or alternatively even if they are part of only one top-level feature. In this case a TrafficArea or TrafficSpace is clearly assigned to exactly one Road, Railway, Track or Waterway object. CityGML offers the possibility to assign multiple function and usage attributes to the same TrafficArea or TrafficSpace. Figure 2 demonstrates this concept for an intersection. TrafficAreas are colored depending on their respective CityGML function or usage attribute value(s). Each TrafficArea is part of a Section or Intersection.

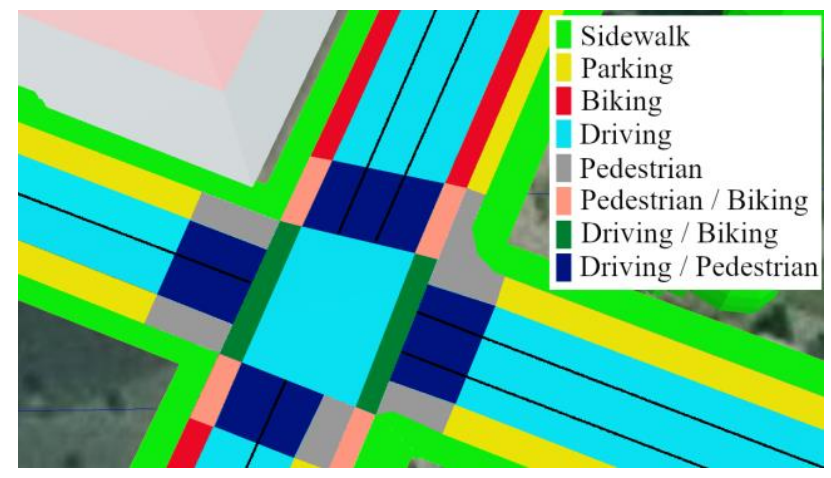

Figure 2. Areal representation of TrafficSpaces colored by function attribute(s)

Similarly, linear representations of TrafficSpaces used by multiple transportation types can be represented with multiple attribute values. Together with predecessor / successor relations this allows (combined) vehicle, bicycle or pedestrian simulations. For linear representations at granularity level 'lane', however, multiple function attributes are most likely not needed, since each transportation type is represented individually. Figure 3 illustrates this idea by showing the schematic linear representation of the same scenario displayed in figure 2. Driving lanes (blue) bicycle lanes (red) and pedestrian sidewalks (green) are modelled with individual linear networks. Parking lanes are not shown here. In order to ensure a consistent representation of linear and areal models, linear segments should also be split at borders of TrafficSpaces with areal representation. This concept is illustrated in the following paragraph.

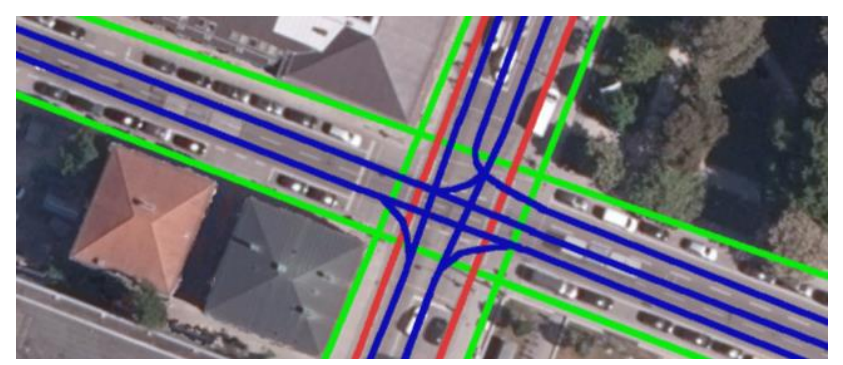

Figure 3. Linear representation of TrafficSpaces colored by function attribute 


\subsection{Modelling multiple Transportation modes within different top-level features}

Level crossings are part of Road as well as Railway infrastructure. Thus, TrafficSpaces that are part of level crossings should be linked to both feature types. Figure 4 shows an instance diagram of the scenario displayed in figure 5 . While this example explains an intersection of Road and Railway objects, this concept also applies to intersections of other transportation types such as Track or Waterway. This way ford crossings could be modelled. Beil \& Kolbe (2017) describe a linking concept for a non-redundant representation of geometries shared by multiple objects at the same time. The given example shows the intersection of a $\operatorname{Road} X$ and a Railway Y at a level crossing. Roads as well as Railways can be segmented into multiple Sections and Intersections, which again are split into individual TrafficSpaces and AuxiliaryTrafficSpaces. In the given example Road X consists of Section A, Section B and Intersection E, while Railway Y consists of Section C, Section D and also Intersection E. It is indicated that Intersection $\mathrm{E}$ is shared by Road $\mathrm{X}$ and Railway $\mathrm{Y}$ by linking both features to Intersection $\mathrm{E}$, thus avoiding redundancies on the geometry level as well as the semantic level.

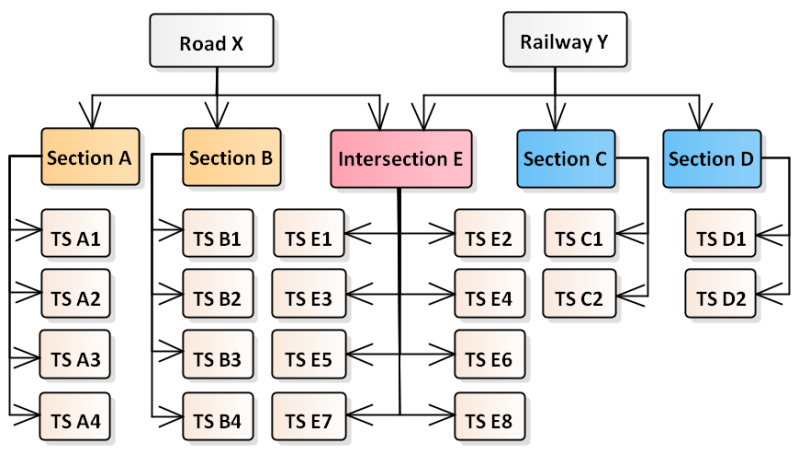

Figure 4. Instance diagram of a level crossing (Intersection of Road and Railway objects)

Each Section and Intersection can be segmented into multiple TrafficSpaces and AuxiliaryTrafficSpaces. Figure 5 illustrates this with an areal representation of a level crossing.

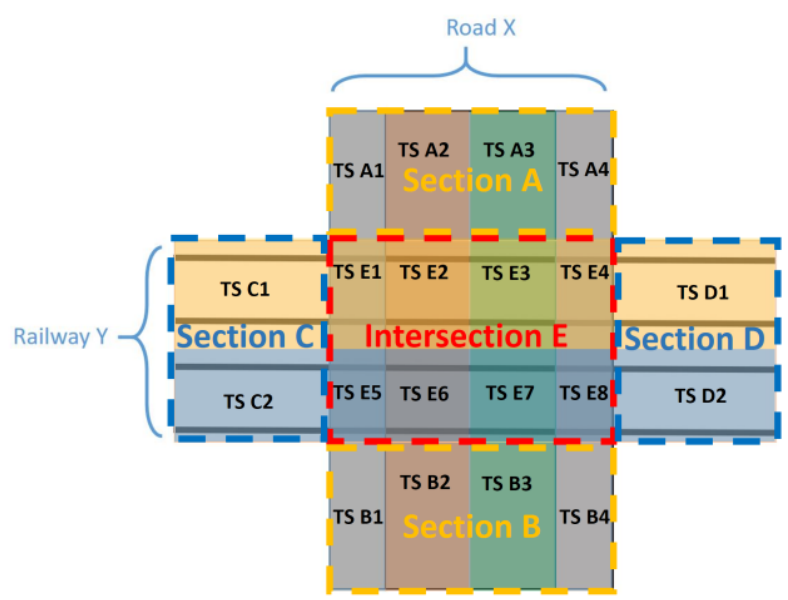

Figure 5. Level crossing represented using TrafficSpaces with areal geometry representation

In the given example Section A consist of four TrafficSpaces, each further specified with CityGML function attributes (two sidewalks and two driving lanes). Similar to the concept presented in chapter 4.2, TrafficSpaces within Intersection E can have multiple function attributes. TrafficSpace 'TS E1', for example, would contain function attributes such as 'sidewalk' as well as 'railway track' since it can be used by both transportation types. Figure 6 shows a linear representation of the same level crossing scenario also in level of granularity 'lane'. As mentioned before, intersecting GM_MultiCurve geometries can be continuous without adding nodes. In this case, lines representing driving lanes or sidewalks intersecting lines representing railway tracks are not split, because traffic members are not allowed to switch between the different transportation types. Lines should be split when representing different TrafficSpaces, in order to ensure consistency between areal and linear representations. This is indicated with small dashes at the end of each line segment. Note that in contrast to the areal representation each TrafficSpace within Intersection E is represented by two linear representations (one for each transportation type).

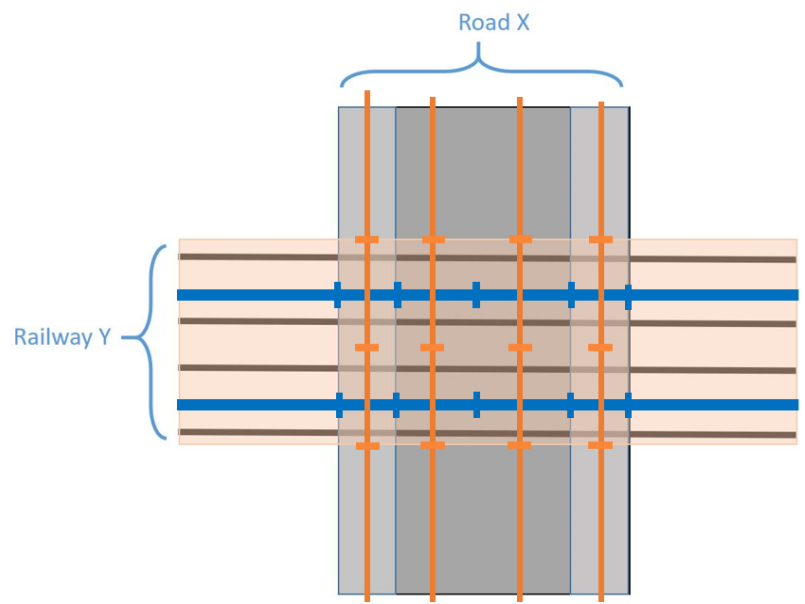

Figure 6. Level crossing using TrafficSpaces with linear geometry representation

\subsection{How to handle shared areas (e.g. Tram / Road)}

Another common scenario, especially within cities, are tramways within road surfaces. In this case, the same physical surface is part of a road and a railway network. This could be modelled in several ways. The first option would be a redundant geometric representation by modelling the same transportation space with overlapping surfaces for Road (used by cars) and Railway (used by trams). This, however, is a redundant representation of reality. Figure 7 illustrates a solution to this problem. The image shows an intersection shared by Road X and Road Y, while part of Road X is simultaneously shared by a Tramway (Railway Z). In this case, a two-level link concept can be applied. Figure 8 shows an instance diagram to illustrate this example. The concept of linking Intersections belonging to multiple Roads is the same as presented earlier. In addition, TrafficSpaces can also be part of multiple Sections or Intersections. In this example, Road $\mathrm{X}$ consists of Section A1, Section B1 and Intersection E1. Road Y consists of Section C1, Section D1 and also Intersection E1. The Tramway sharing surfaces of Road X consist of Section A2, Section B2 and Intersection E2. The new part of this concept is that Sections and Intersection also have links relating them to shared TrafficSpaces. This way a redundant representation of TrafficSpaces can be avoided. Each TrafficSpace can have multiple function and usage attributes. Linear models in granularity 'lane' can avoid this problem by individual 
representations for each transportation type. These concepts can also be applied for combinations of other transportation types such as a ford (intersection of Road and Waterway).

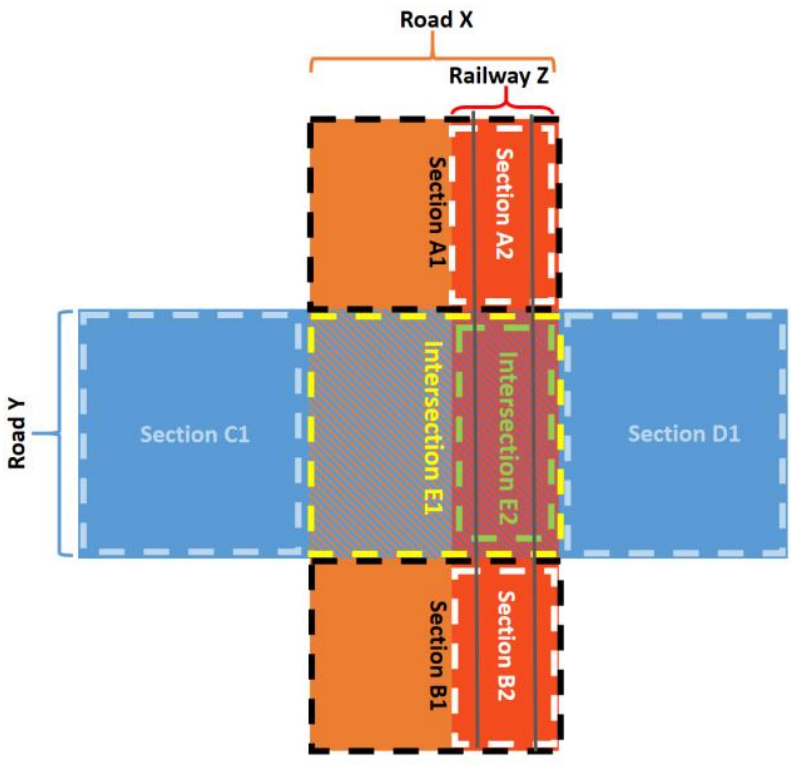

Figure 7. Intersection of two Roads, where one Road contains a Tram (Railway)

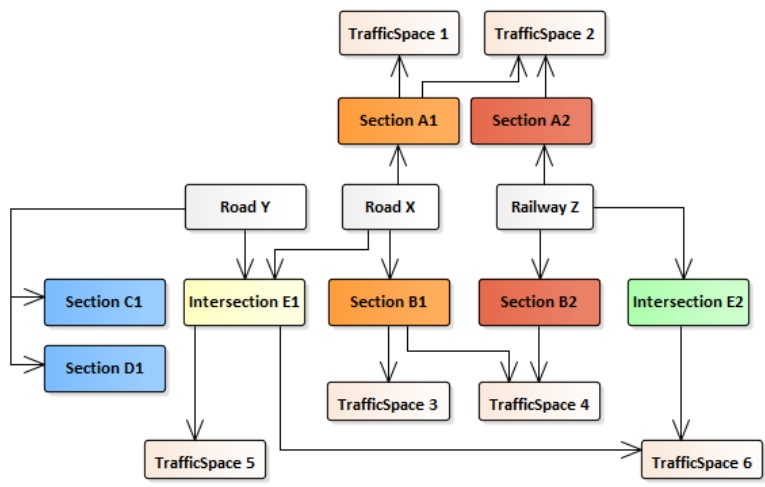

Figure 8. Instance diagram of a two-level linking concept for representing shared surfaces

\section{EXAMPLES AND APPLICATIONS}

In order to illustrate as well as to validate the feasibility of the presented concepts presented in this paper, demos and example datasets have been created. While more and more cities conduct efforts for gathering data on all parts of a city, including streets and railways, to be used in the context of digital twins, there is very little data (openly) available for creating detailed and semantically accurate multimodal street space models. Data gathered with mobile mapping systems could be used for creating models with lane level accuracy. In the context of land management and maintenance areal, semantically accurate models of different transportation infrastructure are important. Information such as pavement rating, area in square meter and name of a specific road for example need to be queried accurately for large regions (such as a continuous road) in order to be able to use this information for calculations such as potential repair costs. For this calculation, areas shared by multiple transportation types need to be taken into account without counting them multiple times. Accurate semantic streetspace models can also be relevant in the context of autonomous driving as they can serve as ground truth (Schwab
\& Kolbe 2019). Information on parts of road surfaces that can also be accessed by pedestrians can be used to identify potential conflict points. Figure 9 shows a CityGML streetspace model generated from OpenDRIVE data. Each TrafficArea contains CityGML function attributes such as 'driving lane', 'crosswalk' or 'bike lane' as well as an attribute indicating corresponding OpenDRIVE reference lines. This way functional information and non-redundant topographical surfaces are combined in a single model and usable for several applications. The bottom image of figure 9 shows the result of a query for all TrafficAreas corresponding to a specific OpenDRIVE reference line (independent of individual functions, highlighted in yellow). Schwab et al. (2020) show how information on areal TrafficAreas with multiple function attributes can be used for combined pedestrian and vehicle simulations. This and other streetspace model demos are visualized using the 3DCityDB Web-Map-Client (Yao et al. 2018) and are provided for interactive exploration on a corresponding website (http://go.tum.de/300369).

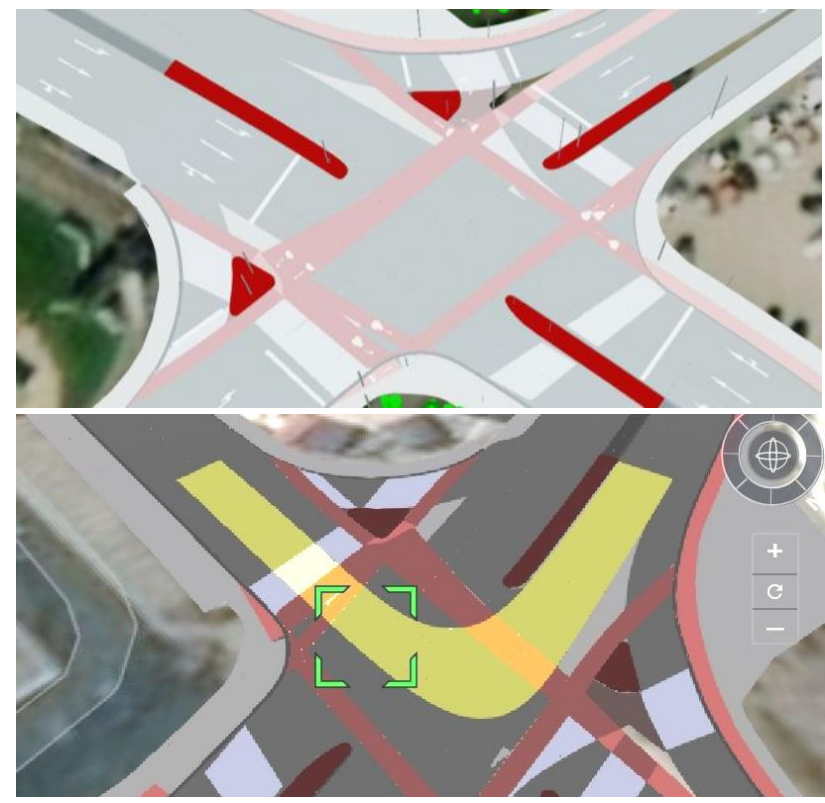

Figure 9. Non-redundant areal representation of multiple transportation types in lane level accuracy

While the new version of CityGML is about to be finalized, the software FME can already be used for generating CityGML 3.0 compliant data by adding a corresponding .xsd file (GML application schema) to the general GML writer.

\section{CONCLUSIONS AND OUTLOOK}

The concepts and examples presented in this paper can be beneficial for a number of applications. Data gathered for the generation of digital twins needs to be structured semantically, geometrically and topologically in order to be useful for a variety of analyses and simulations. Redundancies need to be avoided, because this can lead to misinterpretations, wrong simulation and analyses results and also visualization problems (z-fighting). While an integrated modelling of different infrastructure types is supported by several standards, there are few studies on limitations or best practice examples concerning this topic. Integration of multiple transportation types has to be addressed regarding both the functional and topographical aspects. It was shown how both of these integration types can be achieved with the upcoming new version 3 of the CityGML 
standard. These concepts are also valid for sub-surface transportation infrastructures. Some concepts already have been implemented in order to generate sample data usable for applications. Other aspects such as the presented predecessor / successor concept have not yet been implemented for a specific data set. This will be important for modelling multimodal transportation. While the concepts presented in this paper allow for an integrated, consistent and standardised representation of multiple transportation infrastructure, this also shifts the complexity of using the data towards the generation of the data. Semantic and geometric segmentations have to be taken into account while gathering the data in order to allow further processing. This can also lead to partitioning the surface of a road into many small parts to meet the requirements presented in this paper. However, information on usability of surfaces for multiple transportation modes such as a pedestrian crosswalk used by cars as well as pedestrians is necessary for applications such as combined vehicle and pedestrian simulations (Schwab et al. 2020). A change of surface materials, attributes such as speed limit or temporary events such as road works would require an even greater fragmentation of surfaces or linear structures into small segments. A solution to this problem could be the integration of a linear referencing concept. While the focus on models of buildings has begun to shift towards other thematic parts of a city, such as streetspace, there is only little experience with modelling railway infrastructure in the context of digital 3D city models. Recent developments in projects such as IFCRoad or IFCRailway in other domains such as Building Information Modeling (BIM) indicate the relevance of this topic.

\section{ACKNOWLEDGEMENTS}

The authors would like to thank Ordnance Survey GB (www.ordnancesurvey.co.uk) and 1Spatial (www.1spatial.com/) for sponsoring the publication of this paper.

\section{REFERENCES}

ASAM, 2020. ASAM OpenDRIVE - Open Dynamic Road Information for Vehicle Environment, Version 1.6. Association for Standardisation of Automation and Measuring Systems e.V. https://www.asam.net/standards/detail/opendrive/ (June 2020)

Batty, M., 2018. Digital twins. Environment and Planning B. Urban Analytics and City Science, 45(5), 817-820. doi.org/10.1177/2399808318796416

Beil, C., Kolbe, T. H., 2017. CityGML and the streets of New York - A proposal for detailed street space modelling. In: ISPRS Ann Photo-gram Remote Sens. Spat. Inf. Sci., IV-4/W5, 9-16. doi.org/10.5194/isprs-annals-IV-4-W5-9-2017

Gnatz, S., 2018. Automatisierte Generierung hochdetaillierter 3D-Verkehrswegemodelle für Straßenbahnsimulatoren. Technical University of Munich, Chair of Geoinformatics, Master's Thesis (in German).

Gonzales, E. J., Geroliminis, N., Cassidy, M. J., Daganzo, C. F. 2010. On the allocation of city space to multiple transport modes. In: Transportation planning and technology, 33(8), 643-656. doi.org/10.1080/03081060.2010.527171

Gröger. G., Kolbe, T. H., Nagel, C., Häfele, K-H., 2012. Open Geospatial Consortium (OGC) City Geography Markup Language (CityGML) Encoding Standard, OGC 12-019.
Gröger, G., Plümer, L., 2011. How to achieve consistency for 3D city models. GeoInformatica, 15(1), 137-165. doi.org/10.1007/s10707-009-0091-6

Gruler, H-.C., Stubkjaer, E., Axelsson, P., Wikstrom, L., 2016. OGC Land and Infrastructure Conceptual Model Standard (LandInfra), OGC 15-111.

Haklay, M., Weber, P., 2008. OpenStreetMap User-generated street maps. IEEE Pervasive Computing 7 (4), 12-18.

ISO 14825, 2011. Intelligent transport systems - Geographic Data Files (GDF) - GDF5.0. International Standard, ISO.

ISO 16739-1, 2018. Industry Foundation Classes (IFC) for data sharing in the construction and facility management industries.

INSPIRE, 2014. Thematic Working Group Transport N.: D2.8.I.7 Data Specification on Transport Networks - Technical Guidelines. Version 3.2.

Kutzner, T., Chaturvedi, K., Kolbe, T. H., 2020. CityGML 3.0: New Functions Open Up New Applications. PFG - Journal of Photogrammetry, Remote Sensing and Geoinformation Science, 1-19. doi.org/10.1007/s41064-020-00095-Z

Labetski, A., van Gerwen, S., Tamminga, G., Ledoux, H., Stoter, J., 2018. A proposal for an improved transportation model in CityGML. ISPRS Annals of the Photogrammetry, Remote Sensing and Spatial Information Sciences. XLII-4/W10; 89-96. doi.org/10.5194/isprs-archives-XLII-4-W10-89-2018

Löwner, M. O., Gröger, G., Benner, J., Biljecki, F., Nagel, C., 2016. Proposal for a new lod and multi-representation concept for CityGML. In: ISPRS Annals of Photogrammetry, Remote Sensing \& Spatial Information Sciences, IV-2/W1, 3-12. doi.org/10.5194/isprs-annals-IV-2-W1-3-2016

Müller, P., Wonka, P., Haegler, S., Ulmer, A., Van Gool, L., 2006. Procedural modeling of buildings. In: ACM SIGGRAPH 2006 Papers, 25(3), 614-623.

Schwab, B., Beil, C., Kolbe, T. H., 2020. Spatio-Semantic Road Space Modeling for Vehicle-Pedestrian Simulation to Test Automated Driving Systems. Sustainability, 12(9), 3799. doi.org/10.3390/su12093799

Schwab, B., Kolbe, T. H., 2019. Requirement analysis of 3D road space models for automated driving. In: ISPRS Ann. of Photogramm., Rem. Sens. \& Sp. Inf. Sci., IV-4/W8. 99-106. doi.org/10.5194/isprs-annals-IV-4-W8-99-2019

Tamminga, G. F., 2019. A Novel Design of the Transport Infrastructure for Traffic Simulation Models. Trail. Ph.D. Thesis, Delft University of Technology.

Yao, Z., Nagel, C., Kunde, F., Hudra, G., Willkomm, P., Donaubauer, A., Adolphi, T., Kolbe, T. H., 2018. 3DCityDB - a 3D geodatabase solution for the management, analysis, and visualization of semantic 3D city models based on CityGML. Open Geospatial Data, Software and Standards, 3(1), 1-26. doi.org/10.1186/s40965-018-0046-7 